GOTERM_BP_ALL

Term Hits PValue (b) Benjamini (c)

GOTERM_BP_ALL

GO:0048856 anatomical structure development

GOTERM BP ALL

GO:0048731 system development

88 1.48 E-10 $1.94 \mathrm{E}-7$

GOTERM BPALL

Functional Group 2

Category

GO:0007275 multicellular organismal development

GOTERM BP ALL

GOTERM_BP_ALL

Enrichment score: 7.06

\begin{tabular}{l|l|l|l}
\hline Term & Hits PValue Benjamin
\end{tabular}

$1063.83 \mathrm{E}-10-3.36 \mathrm{E}-7$

GO:0001568 blood vessel development

Hits PValue Benjamini

\begin{tabular}{|l|l|l|l|}
\hline GOTERM_BP_ALL & GO:0048514 blood vessel morphogenesis & $191.63 \mathrm{E}-7$ & $5.04 \mathrm{E}-5$
\end{tabular}

\begin{tabular}{l|l|l|l|l|}
\hline GOTERM BP_ALL & GO:0048646 anatomical structure formation & 19 & $5.08 \mathrm{E}-7$ & $1.48 \mathrm{E}-4$
\end{tabular}

\begin{tabular}{|l|l|l|l|l|l|l}
\hline GOTERM_BP_ALL & GO:0001525 angiogenesis & $175.21 \mathrm{E}-7$ & $1.44 \mathrm{E}-4$
\end{tabular}

Functional Group 3

Category

SP_PIR_KEYWORDS

GOTERM_CC_ALL

Enrichment score: 6.79

Term

Hits PValue Benjamini

\begin{tabular}{l|l|l|l|l}
\hline extracellular matrix & 23 & $1.39 \mathrm{E}-8$ & $2.96 \mathrm{E}-6$
\end{tabular}

\begin{tabular}{|l|l|l|l|l|}
\hline GO:0005578 proteinaceous extracellular matrix & 27 & $4.61 \mathrm{E}-7$ & $1.33 \mathrm{E}-4$
\end{tabular}

\begin{tabular}{|l|l|l|l|l|}
\hline GOTERM_CC_ALL & GO:0031012 extracellular matrix & 27 & $6.44 \mathrm{E}-7$ & $1.39 \mathrm{E}-4$ \\
\hline
\end{tabular}

\begin{tabular}{l|l|l|l|l} 
Functional Group 4 & Enrichment score: 5.84 & & \\
\hline Category & Term & Hits & PValue & Benjamini
\end{tabular}

\begin{tabular}{|l|l|l|l|l|l|l|l}
\hline GOTERM_BP_ALL & GO:0048534 hemopoietic or lymphoid organ & $20.35 \mathrm{E}-7$ & $2.45 \mathrm{E}-4$ \\
\hline
\end{tabular}

\begin{tabular}{l|l|l|l|l}
\hline GOTERM_BP_ALL & GO:0030097 hemopoiesis & 19 & $1.42 \mathrm{E}-6$ & $3.41 \mathrm{E}-4$
\end{tabular}

\begin{tabular}{|l|l|l|l|l|}
\hline GOTERM_BP_ALL & GO:0002520 immune system development & 20 & $2.19 \mathrm{E}-6$ & $5.01 \mathrm{E}-4$ \\
\hline
\end{tabular}

\begin{tabular}{l|l|l|l|l}
\hline Functional Group 5 & Enrichment score: 5.76 & & & \\
\hline Category & Term & Hits & PValue & Benjamini
\end{tabular}

\begin{tabular}{l|l|l|l|l|l}
\hline Category & Term & Hits & PValue & Benjamini \\
\hline SP_PIR_KEYWORDS & triple helix & $112.84 \mathrm{E}-9$ & $1.01 \mathrm{E}-6$
\end{tabular}

\begin{tabular}{l|l|l|l}
\hline SP_PIR_KEYWORDS & hydroxylysine & $106.70 \mathrm{E}-8$ & $8.92 \mathrm{E}-6$ \\
\hline
\end{tabular}

\begin{tabular}{l|l|l|l|}
\hline SP_PIR_KEYWORDS & hydroxyproline & $101.15 \mathrm{E}-7$ & $1.36 \mathrm{E}-5$
\end{tabular}

IPR008160: Collagen triple helix repeat

\begin{tabular}{|l|l|l|l|}
\hline INTERPRO & IPR008161:Collagen helix repeat & $116.05 \mathrm{E}-7$ & 0.0017 \\
\hline
\end{tabular}

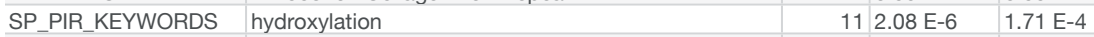

\begin{tabular}{l|l|l|l|l}
\hline GOTERM_BP_ALL & GO:0006817 phosphate transport & 13 & $3.49 \mathrm{E}-6$ & $6.54 \mathrm{E}-4$
\end{tabular}

\begin{tabular}{|l|r|r|r|}
\hline SP_PIR_KEYWORDS & collagen & $125.54 \mathrm{E}-5$ & 0.0036
\end{tabular}

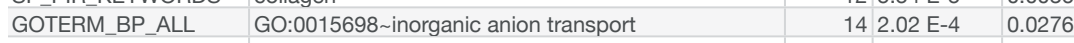

\begin{tabular}{l|l|l|l|l|}
\hline GOTERM_BP_ALL & GO:0006820 anion transport & 15 & $4.35 \mathrm{E}-4$ & 0.0570
\end{tabular}

\title{
repressed genes
}

Functional Group 1

Category

SMART

INTERPRO

PIR_SUPERFAMILY

INTERPRO

Functional Group 2

Category

Enrichment score: 6.24

Term

IPR000558:Histone H2B

PIRSF002050:histone H2B

\begin{tabular}{|l|l|l|l|}
\hline PIRSF002050:histone H2B & $74.72 \mathrm{E}-7$ & 0.001 \\
\hline IPR009072:Histone-fold & 9 & $6.66 \mathrm{E}-6$ & 0.013 \\
\hline
\end{tabular}

Hits

PValue Benjamini

$71.65 \mathrm{E}-7 \quad 1.00 \mathrm{E}-4$

\begin{tabular}{l|l|l}
7 & $1.95 \mathrm{E}-7$ & $5.79 \mathrm{E}-4$
\end{tabular}

Enrichment score: 5.24

Term

E-6 0.013

GOTERM BP ALL

GOTERM_BP_ALL

\begin{tabular}{l|r|l|l}
\hline Term & Hits & PValue & Benjamini \\
\hline GO:0006915 apoptosis & 40 & $1.30 \mathrm{E}-6$ & $7.60 \mathrm{E}-4$
\end{tabular}

GOTERM_BP_ALL

\begin{tabular}{l|l|l|l|l}
\hline GO:0012501 programmed cell death & 40 & $1.62 \mathrm{E}-6$ & $8.55 \mathrm{E}-4$
\end{tabular}

GOTERM BP ALL

\begin{tabular}{|l|l|l|l|}
\hline Functional Group 3 & Enrichment score: 5.14 & \\
\hline
\end{tabular}

Category Term Hits PValue Benjamini

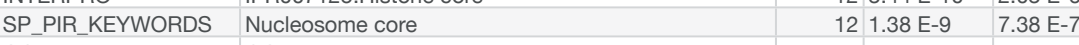

\begin{tabular}{|l|l|l|l|l|}
\hline GOTERM_BP_ALL & GO:0006334 nucleosome assembly & $135.01 \mathrm{E}-7$ & $4.39 \mathrm{E}-4$ \\
\hline
\end{tabular}

GOTERM CC_ALL GO:0000786 nucleosome

\begin{tabular}{l|l|r|l|l|}
\hline GOTERM_BP_ALL & GO:0031497 chromatin assembly & $132.21 \mathrm{E}-6$ & 0.0010
\end{tabular}

\begin{tabular}{|l|r|r|r|}
\hline INTERPRO & IPR009072:Histone-fold & $96.66 \mathrm{E}-6$ & 0.0130 \\
\hline
\end{tabular}

\begin{tabular}{|l|l|r|l|l|l|l|l|l|l|}
\hline GOTERM_BP_ALL & GO:0006333 chromatin assembly or disassembly & 14 & $1.74 \mathrm{E}-5$ & 0.0057
\end{tabular}

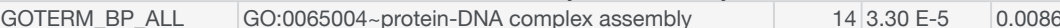

\begin{tabular}{l|l|r|l}
\hline SP_PIR_KEYWORDS & Chromosomal protein & $127.27 \mathrm{E}-5$ & 0.0077 \\
\hline
\end{tabular}

\begin{tabular}{l|l|l|l|l|l}
\hline GOTERM_CC_ALL & GO:0000785 chromatin & $14.76 \mathrm{E}-4$ & 0.0194
\end{tabular}

\begin{tabular}{l|l|l|l|l}
\hline GOTERM_BP_ALL & GO:0006323 DNA packaging & 18 & $7.10 \mathrm{E}-4$ & 0.0936
\end{tabular}

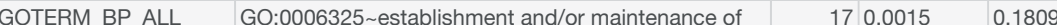

\begin{tabular}{|l|l|l|l|l|}
\hline GOTERM_BP_ALL & GO:0051276 chromosome organization and & 190.0026 & 0.2339 \\
\hline
\end{tabular}

\begin{tabular}{l|l|l|l|l}
\hline Functional Group 4 & Enrichment score: 4.51 & & \\
\hline Category & Term & Hits & PValue & Benjamini
\end{tabular}

\begin{tabular}{l|l|r|l|l}
\hline Category & Term & Hits & PValue & Benjamin \\
\hline GOTERM_CC_ALL & GO:0043229 intracellular organelle & 228 & $1.09 \mathrm{E}-5$ & 0.0010
\end{tabular}

\begin{tabular}{|l|l|l|l|l|}
\hline GOTERM_CC_ALL & GO:0043226 organelle & 228 & $1.14 \mathrm{E}-5$ & $9.91 \mathrm{E}-4$ \\
\hline
\end{tabular}

\begin{tabular}{ll|l|l|l|}
\hline GOTERM_CC_ALL & GO:0043231 intracellular membrane-bound & $1998.33 \mathrm{E}-5$ & 0.0055
\end{tabular}

\begin{tabular}{l|l|l|l}
\hline GOTERM_CC_ALL & GO:0043227 membrane-bound organelle & $1998.58 \mathrm{E}-5$ & 0.0053
\end{tabular}

\begin{tabular}{|l|l|l|l|l|}
\hline Functional Group 5 & Enrichment score: 4.33 \\
\hline
\end{tabular}

\begin{tabular}{l|l|l|l} 
Category & Term & Hits PValue Benjamin
\end{tabular}

\begin{tabular}{|l|r|r|r|}
\hline GOTERM_CC_ALL & GO:0043292 contractile fiber & $102.36 \mathrm{E}-5$ & 0.0018 \\
\hline
\end{tabular}

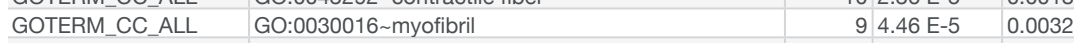

\begin{tabular}{l|l|l|l|l|l|l|l}
\hline GOTERM_CC_ALL & GO:0044449 contractile fiber part & 9 & $9.176 \mathrm{E}-5$ & 0.0052
\end{tabular}

${ }^{a}$ Enrichment score is the negative log of geometric mean of each member's P-value in the cluster. bP-value is calculated by Fisher's exact tests.

'Benjamini-Hochberg is the P-value corrected for multiple comparisons. 